\title{
Axial Alignment of the Lower Extremity in Chinese Adults"
}

\author{
BY W. M. TANG, F.R.C.S.E.†, Y. H. ZHU, M.D. $\dagger$, AND K. Y. CHIU, F.R.C.S.E. $\dagger$ \\ Investigation performed at the Division of Joint Replacement Surgery, \\ Department of Orthopaedic Surgery, Queen Mary Hospital, Pokfulam, Hong Kong, China
}

\begin{abstract}
Background: The restoration of normal axial alignment of the lower extremity is important to surgeons who perform reconstructive surgery of the knee. However, data on the normal alignment of the lower extremity in Chinese adults are not available.

Methods: The axial alignment of the lower extremity in twenty-five adult male and twenty-five adult female volunteers of southern Chinese origin was measured on weight-bearing radiographs of the entire lower limb. The mean age was twenty-four years for the male volunteers and twenty-three years for the female volunteers. The results were compared with those of two similar studies of white volunteers in the United States.

Results: The medial inclination of the tibial plateau in the Chinese subjects (mean and standard deviation, $5.4 \pm 2.5$ degrees for women and $4.9 \pm 2.3$ degrees for men) was greater than the commonly reported 3 degrees. The extremities of the Chinese women were found to have a mean of $2.2 \pm 2.5$ degrees of varus alignment, and those of the Chinese men had a mean of $2.2 \pm 2.7$ degrees of varus alignment.

Conclusions: Compared with the white subjects described in the studies by Moreland et al. and Hsu et al., the Chinese subjects had significantly larger medial inclination of the knee joint (knee-joint obliquity) $(p<0.005)$ and the female Chinese subjects had significantly more varus alignment of the lower extremity $(\mathrm{p}<\mathbf{0 . 0 2 5})$.

Clinical Relevance: Five degrees of external rotation of the femoral component, instead of the commonly reported 3 degrees, may be required to obtain a rectangular flexion gap in total knee arthroplasty in Chinese patients. The racial difference in the knee-joint obliquity may contribute to the racial difference in the ratio of knee osteoarthritis to hip osteoarthritis. Additional studies are necessary to confirm this relationship.
\end{abstract}

\footnotetext{
* No benefits in any form have been received or will be received from a commercial party related directly or indirectly to the subject of this article. Funds were received in total or partial support of the research or clinical study presented in this article. The funding source was a research grant from the Committee on Research and Conference Grants, University of Hong Kong.

$\dagger$ Division of Joint Replacement Surgery, Department of Orthopaedic Surgery, Queen Mary Hospital, 102 Pokfulam Road, Pokfulam, Hong Kong, China. E-mail address: wmtanga@hkucc.hku.hk (W. M. Tang).
}

Copyright () 2000 by The Journal of Bone and Joint Surgery, Incorporated
Achieving normal axial alignment of the lower extremity is important to surgeons who perform reconstructive surgery of the knee, such as total knee arthroplasty and high tibial osteotomy. The importance of restoring the axial alignment of the lower extremity following total knee arthroplasty has been previously emphasized ${ }^{9}$. A total knee replacement with varus alignment has been shown to fail substantially earlier than those with neutral or valgus alignment ${ }^{13}$.

The normal alignment of the lower extremity has been documented by Moreland et al. ${ }^{12}$ and Hsu et al. with use of radiographs of the lower extremity (Table I). To the best of our knowledge, no similar investigations have been performed for Chinese adults.

To establish the so-called norm for southern Chinese adults, and to identify the difference between white and Chinese individuals with respect to the axial alignment of the lower extremity, we performed a radiographic investigation of the lower extremities of twenty-five men and twenty-five women of southern Chinese origin.

\section{Materials and Methods}

Twenty-five male and twenty-five female volunteers of southern Chinese origin were evaluated. None of the subjects had knee pain, a history of knee trauma, or obvious knee deformity. The mean age was twenty-four years (range, twenty-two to thirty-one years) for the men and twenty-three years (range, twenty-one to twenty-nine years) for the women. All of the subjects were asked to stand, wearing their usual footwear, with both knees fully extended and both patellae facing forward ${ }^{8}$. A weight-bearing anteroposterior radiograph of the entire lower limb then was made with the $\mathrm{x}$-ray beam centered at the knees at a distance of 2.5 meters. A cassette holding long radiographs (300 by 900 millimeters) was placed immediately behind the subject. Both lower extremities were included in one radiograph for each subject. A lead shield was used to protect the gonads. A setting of sixty milliampere-seconds and a kilovoltage of sixty-five to eighty were required to provide the necessary exposure.

The axial alignment was measured according to the methods described by Moreland et al. ${ }^{12}$, with slight modifications. The centers of the femoral head, the knee, and the ankle first were identified. The center of the femoral head was determined with use of Mose circles. The center of the knee was selected as the midpoint of four measured points: (1) the center of the tibia at the level of the subchondral bone of the medial tibial plateau, (2) the midpoint between the tips of the tibial spines, (3) the midpoint between the femoral condyles at the level of the subchondral bone of the lateral femoral condyle, and (4) the center of the femoral intercondylar notch at the trough of the femoral notch (Fig. 1). Moreland et al. used soft-tissue edges to define a fifth reference point, but this was not used in our study because the edge of the soft tissue cannot be located as accurately as the osseous tissue can. The center of the ankle was a point midway between two 
TABLE I

Comparison of KneE-Angle Measurements in Chinese and White Adults*

\begin{tabular}{|c|c|c|c|c|c|c|}
\hline \multirow[b]{2}{*}{ Angle } & \multicolumn{2}{|c|}{$\begin{array}{l}\text { Series of Moreland } \\
\text { et al. } .^{12} \dagger \text { (degrees) }\end{array}$} & \multicolumn{2}{|c|}{$\begin{array}{l}\text { Present Series } \neq \\
\text { (degrees) }\end{array}$} & \multirow{2}{*}{$\begin{array}{c}\text { Series of Hsu } \\
\text { et al. } \$ \text { (degrees) } \\
\text { Right and Left }\end{array}$} & \multirow{2}{*}{$\begin{array}{c}\begin{array}{c}\text { Present Series } \\
\text { (degrees) }\end{array} \\
\text { Right and Left }\end{array}$} \\
\hline & Right & Left & Right & Left & & \\
\hline \multicolumn{7}{|l|}{ Men } \\
\hline A & $178.5 \pm 2.0$ & $178.9 \pm 2.1$ & $\begin{array}{c}177.8 \pm 2.5 \\
(p>0.25)\end{array}$ & $\begin{array}{c}177.8 \pm 2.9 \\
(\mathrm{p}>0.1)\end{array}$ & $177.7 \pm 2.3$ & $\begin{array}{l}177.8 \pm 2.7 \\
(p>0.25)\end{array}$ \\
\hline B & $93.0 \pm 1.6$ & $92.6 \pm 1.4$ & $\begin{array}{r}94.7 \pm 2.5 \\
(\mathrm{p}<0.005)\end{array}$ & $\begin{array}{r}95.0 \pm 2.2 \\
(\mathrm{p}<0.005)\end{array}$ & $91.0 \pm 1.4$ & $\begin{array}{r}94.9 \pm 2.3 \\
(\mathrm{p}<0.005)\end{array}$ \\
\hline $\mathrm{C}$ & $5.8 \pm 0.7$ & $6.0 \pm 1.0$ & $\begin{array}{c}5.6 \pm 0.9 \\
(\mathrm{p}>0.25)\end{array}$ & $\begin{array}{l}5.7 \pm 0.8 \\
(\mathrm{p}>0.1)\end{array}$ & & $5.6 \pm 0.8$ \\
\hline $\mathrm{D}$ & $4.0 \pm 0.7$ & $4.1 \pm 0.9$ & $\begin{array}{l}3.7 \pm 0.9 \\
(p>0.1)\end{array}$ & $\begin{array}{l}3.5 \pm 0.8 \\
(\mathrm{p}>0.1)\end{array}$ & $4.4 \pm 1.7$ & $\begin{array}{r}3.6 \pm 0.8 \\
(p<0.005)\end{array}$ \\
\hline $\mathrm{E}$ & $90.7 \pm 3.2$ & $89.6 \pm 2.3$ & $\begin{array}{l}91.6 \pm 3.3 \\
(p>0.25)\end{array}$ & $\begin{array}{r}91.2 \pm 3.1 \\
(\mathrm{p}<0.025)\end{array}$ & & $91.4 \pm 3.1$ \\
\hline \multicolumn{7}{|l|}{ Women } \\
\hline A & & & $178.1 \pm 2.8$ & $177.5 \pm 2.3$ & $178.7 \pm 1.8$ & $\begin{array}{l}177.8 \pm 2.5 \\
(\mathrm{p}<0.025)\end{array}$ \\
\hline B & & & $95.3 \pm 2.5$ & $95.5 \pm 2.5$ & $90.1 \pm 1.9$ & $\begin{array}{r}95.4 \pm 2.5 \\
(\mathrm{p}<0.005)\end{array}$ \\
\hline $\mathrm{C}$ & & & $5.7 \pm 1.0$ & $5.6 \pm 1.1$ & & $5.7 \pm 1.0$ \\
\hline $\mathrm{D}$ & & & $3.3 \pm 0.5$ & $3.0 \pm 0.6$ & $3.6 \pm 1.1$ & $\begin{array}{r}3.1 \pm 0.6 \\
(\mathrm{p}<0.005)\end{array}$ \\
\hline E & & & $90.7 \pm 2.4$ & $90.9 \pm 2.6$ & & $90.8 \pm 2.5$ \\
\hline
\end{tabular}

*The values are given as the mean and the standard deviation.

$\dagger$ The data are given for twenty-five white men.

\$The data are given for twenty-five male and twenty-five female Chinese subjects.

$\S$ The data are given for thirty male and thirty female white subjects.

reference points: (1) the center of the outer cortex between the two malleoli at the level of the subchondral bone of the distal part of the tibia, and (2) the center of the talus at the level of the subchondral bone of the talus (Fig. 2). For the same reason that soft-tissue edges were not used in locating the center of the knee, they were not used in locating the center of the ankle.

The mechanical axes of the femur and the tibia were the lines joining the center of the knee to the center of the femoral head and the center of the knee to the center of the ankle, respectively. Two additional reference points were used to define the two anatomical axes of the femur. Femoral shaft center I was the point in the middle of the medullary canal at the midpart of the femoral shaft. Femoral shaft center II was located at the middle of the medullary canal at a level ten centimeters proximal to the knee-joint surface (Fig. 3). Femoral anatomical axis I was a line drawn from femoral shaft center I to the center of the knee. Another line was drawn from femoral shaft center I to femoral shaft center II. This line, constructed on the basis of the two femoral shaft centers, followed the femoral medullary canal more closely and was termed femoral anatomical axis II (Fig. 3).

Two additional axes were constructed: (1) the transverse axis of the knee was a line tangential to the most distal points of the femoral condyles, and (2) the transverse axis of the ankle was the extension of the subchondral plate of the distal part of the tibia (Fig. 3).

Five angles were defined. Angle A was the medial angle formed by the mechanical axes of the femur and the tibia. Angle B was the inferolateral angle formed by the transverse axis of the knee and the mechanical axis of the tibia. Angles D and $\mathrm{C}$ were formed by the mechanical axis of the femur and the femoral anatomical axes I and II, respectively. Angle $\mathrm{E}$ was the inferolateral angle formed by the transverse axis of the ankle and the mechanical axis of the tibia. A finely calibrated goniometer was used to measure the angles within an accuracy of 0.5 degree. The length of the femur was measured along the mechanical axis of the femur from the superior border of the femoral head to the center of the knee.

All radiographs were measured independently by two of us
(W. M. T. and Y. H. Z). The centers and the lines constructed by one of us (W. M. T.) were completely erased before the measurements were repeated by the other one. The two sets of data were compared with use of the independent-samples t test, and they were not found to be significantly different from each other $(p>0.8)$. The measurements on all of the radiographs were repeated by the same investigators after an interval of two months. The data from each investigator were compared, with use of the independent-samples t test, with those obtained in the first measurement by the same investigator. No significant difference was found in the measurements made by either investigator $(p>0.7$ for the first investigator and $p>0.8$ for the second).

We compared our results with those in two similar investigations performed on white subjects in the United States by Moreland et al. ${ }^{12}$ and Hsu et al. ${ }^{5}$. In their studies, radiographs of the entire lower extremities were made for each subject. Moreland et al. included only male subjects, and the results of the left and right sides were calculated separately. Hsu et al. included both male and female subjects, but the results on the left and right sides were not calculated separately (Table I). Hsu et al. divided the subjects into two groups according to their age. We compared our results with the younger group of subjects (that is, the twenty-five to forty-year-old group) in the study by Hsu et al. because we included only young subjects. Hsu et al. did not report angles $\mathrm{C}$ and $\mathrm{E}$.

Unless otherwise stated, continuous variables were compared with use of the independent-samples t test.

\section{Results}

The results are summarized in Table I.

Angle A represented the overall alignment of the lower extremity. An extremity in varus alignment has an angle $\mathrm{A}$ of less than 180 degrees. In our study, angle A was a mean (and standard deviation) of $177.8 \pm 2.5$ degrees (range, 173.0 to 182.0 degrees) in the women 


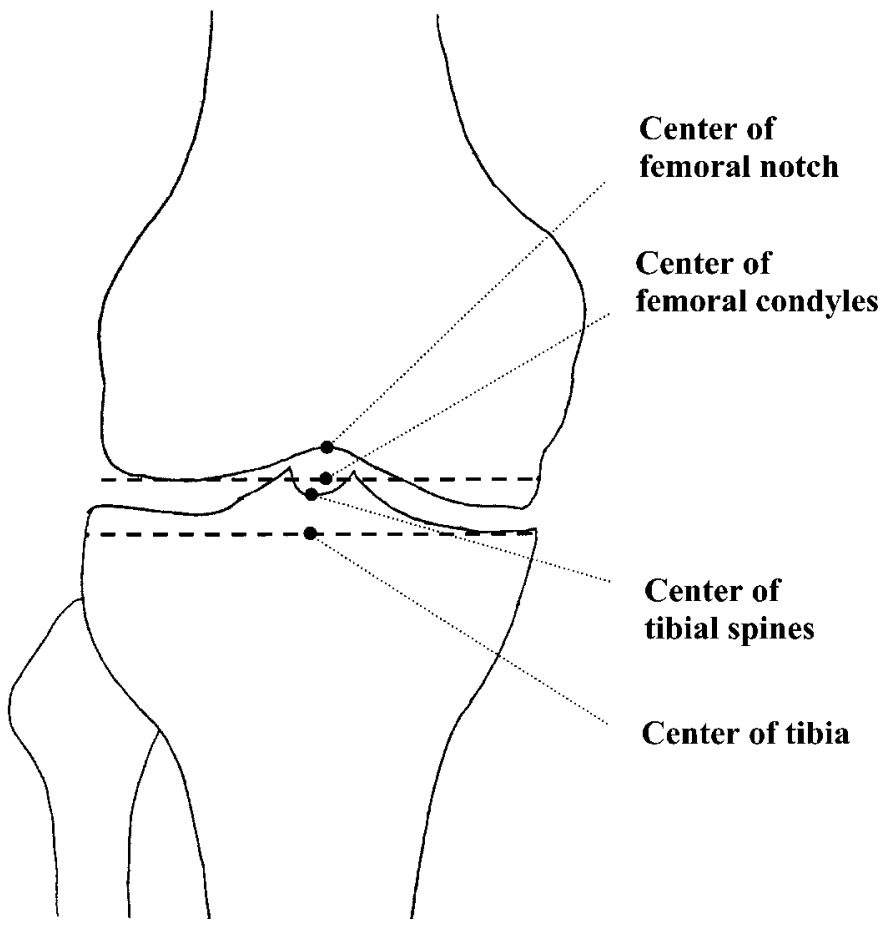

FIG. 1

Drawing showing points used to determine the center of the knee.

and $177.8 \pm 2.7$ degrees (range, 172.0 to 182.5 degrees) in the men. Therefore, the extremities of the Chinese women had a mean of $2.2 \pm 2.5$ degrees of varus alignment, and those of the Chinese men had a mean of $2.2 \pm$ 2.7 degrees of varus alignment. Angle A was significantly smaller than 180 degrees (paired-samples t test, $\mathrm{p}<0.002$ in both genders and on both the left and the right side). Angle A in our male volunteers did not differ significantly from that in the study of white subjects by Moreland et al. ${ }^{12}$ ( $p>0.25$ for the right side and $p>$ 0.1 for the left side). Angle A in our female volunteers, however, was significantly smaller than that in the white female subjects in the study by Hsu et al..$^{5}(\mathrm{p}<0.025)$. The knees of our Chinese female subjects, therefore,

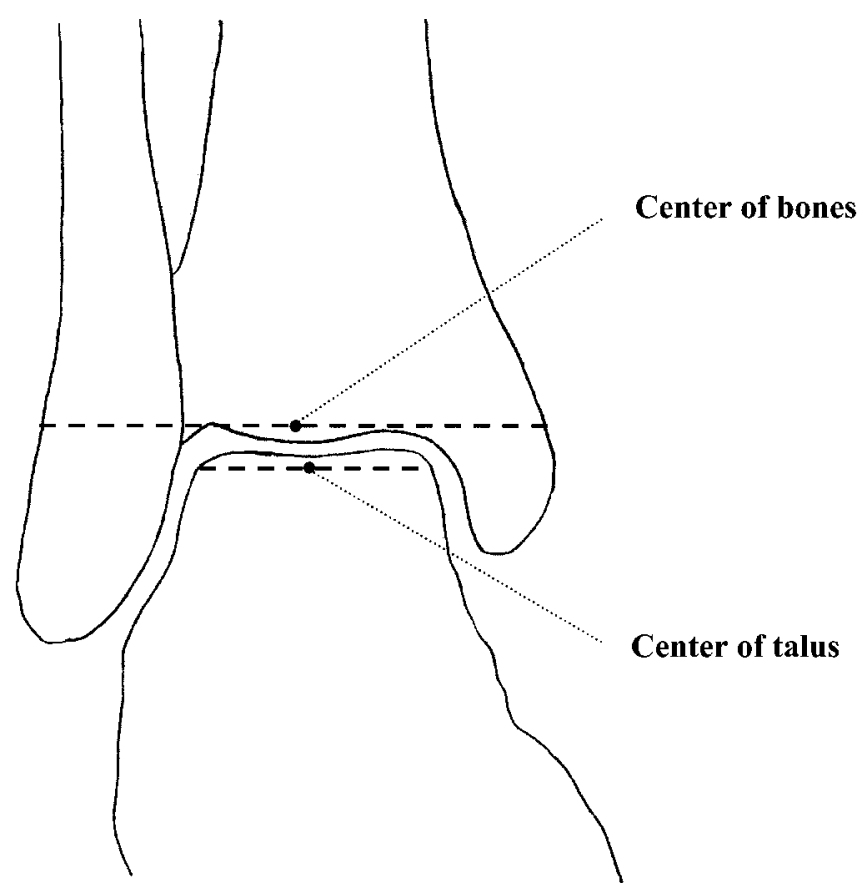

FIG. 2

Drawing showing points used to determine the center of the ankle. 
were in more varus alignment than were those in the white female subjects in the study by Hsu et al. Angle A in the male subjects in our series was not found to be significantly different from that in the male subjects in the study by Hsu et al. $(\mathrm{p}>0.25)$.

Angle B is the inferolateral angle between the kneejoint surface and the mechanical axis of the tibia. The knee-joint surface is perpendicular to the mechanical axis if angle $B$ is 90 degrees. The knee-joint surface is inclined toward the medial side if angle B is larger than 90 degrees. Angle B, therefore, is an index of the obliquity of the knee joint. Angle B was a mean (and standard deviation) of $95.4 \pm 2.5$ degrees (range, 89.0 to 100.0 degrees) in the women and $94.9 \pm 2.3$ degrees (range, 90.5 to 102.0 degrees) in the men in our study. Angle B was significantly larger than 93 degrees (paired-samples $t$ test, $\mathrm{p}<0.001$ in both genders and on both the left and the right side). The mean medial inclination of the knee- joint surfaces in our female and male subjects therefore was $5.4 \pm 2.5$ degrees and $4.9 \pm 2.3$ degrees, respectively, which is significantly more oblique than the commonly reported 3 degrees. Angle B in both the right and the left extremity in our male subjects was significantly larger than that in the white subjects in the study by Moreland et $a^{12}{ }^{12}(\mathrm{p}<0.005$ on both sides). In the study by Hsu et al. ${ }^{5}$, angle B was a mean of $91.0 \pm 1.4$ degrees in thirty white men (sixty lower extremities) who ranged in age from twenty-five to forty years and a mean of $90.1 \pm 1.9$ degrees in thirty white women who ranged in age from twenty-five to forty years. In our Chinese volunteers, angle B was significantly larger than that in the subjects in the study by Hsu et al. ( $\mathrm{p}<0.005$ in both genders).

While angle $\mathrm{D}$ in our male subjects was significantly larger than that in our female subjects on both sides $(\mathrm{p}=$ 0.025 for the right side and $p=0.01$ for the left side), no difference related to gender was found in angles A, B, C,

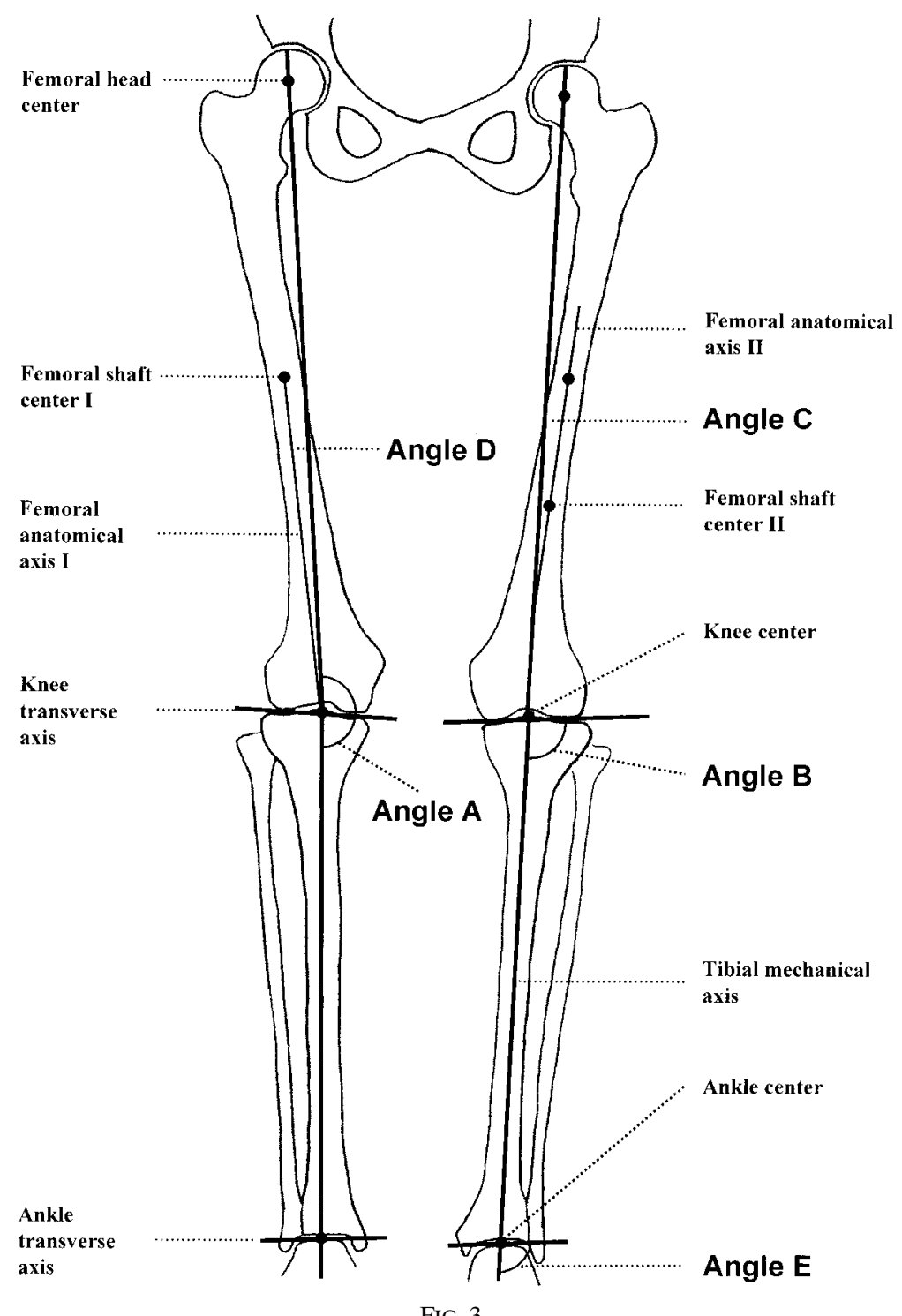

FIG. 3

Drawing showing the axes and the angles. 
or $\mathrm{E}$ in our subjects. No association was found between angle $\mathrm{B}$ and angle $\mathrm{E}$.

Angles C and D in our male subjects were not significantly different from those on either the left or the right side in the subjects described by Moreland et al. ${ }^{12}(\mathrm{p}>0.1$ for both angles and for both sides). Hsu et al. ${ }^{5}$ did not report angle C. Angle D in our subjects was significantly smaller than that in the subjects described by Hsu et al. ( $p<0.005$ for both genders). The length of the femur was a mean (and standard deviation) of $44.8 \pm 1.7$ centimeters (range, 42.0 to 48.4 centimeters) in the female subjects and $47.4 \pm 2.2$ centimeters (range, 42.0 to 51.2 centimeters) in the male subjects in our study. No association was found between the length of the femur and angles $\mathrm{C}$ and $\mathrm{D}$ in either the male or the female subjects.

\section{Discussion}

Orthopaedic operations often involve the correction of a deformity. The so-called normal anatomy of the lower extremity to be restored with total knee arthroplasty has remained an area of controversy ${ }^{5-7,12}$. As noted by Insall, one should be cautious in describing what is "normal" because of the substantial individual variations. Currently, designers of most total knee arthroplasty systems recommend placement of the components in such a way that the transverse axis of the artificial knee joint is perpendicular to the mechanical axes of the tibia and the femur?. The resulting alignment of the lower extremity, therefore, is in close proximity to the alignment documented by Moreland et al. ${ }^{12}$ and Hsu et al. ${ }^{5}$.

The mechanical axes of the femur and the tibia did not form a straight line in either the female or the male Chinese volunteers in our series. This finding is in contrast to the general consensus that has been described previously ${ }^{6,7}$. Our female volunteers had more varus alignment of the knee than did the white female subjects in the study by Hsu et al. ${ }^{5}$. We did not, however, find the same difference between our male subjects and the white male subjects described by Moreland et al. ${ }^{12}$ and Hsu et al.

Insall ${ }^{7}$ and Hungerford et al. ${ }^{6}$ described a 3-degree varus alignment of the knee-joint surface with reference to the mechanical axis of the tibia. Although Moreland et al. ${ }^{12}$ confirmed this 3 -degree varus in white men, the finding was not reproduced in the study by Hsu et al. ${ }^{5}$ In our study of Chinese subjects, the surface of the knee joint had a medial inclination with respect to the tibia that was a mean of 5.4 degrees in the women and 4.9 degrees in the men. The values were found to be significantly different from those of the white subjects in the studies of Moreland et al. and Hsu et al. $(\mathrm{p}<0.005)$. Therefore, if the tibial cut in a total knee arthroplasty is placed perpendicular to the mechanical axis of the tibia $^{7}, 5$ degrees of external rotation of the femoral component instead of the commonly reported 3 degrees might be necessary to produce a rectangular flexion gap in Chinese patients.

The use of an intramedullary guide for the distal femoral cut is currently standard practice in total knee arthroplasty. Most of the instrumentation systems offer a standard 6-degree cutting block to guide the distal femoral cut in order to match the commonly reported 6degree physiological valgus angulation of the femur. The intramedullary guide is best represented in our study by femoral anatomical axis II, which did not intersect the mechanical axis of the femur at the center of the knee. In order to produce a distal femoral cut that is perpendicular to the mechanical axis of the femur - that is, 6 degrees of valgus angulation with respect to femoral anatomical axis II of the femur (angle C) - the entry point of the intramedullary rod should be shifted medially. We recommend a five-millimeter medial shift of the entry hole from the apex of the intercondylar notch. Fang et al. ${ }^{3}$ showed that femora in Chinese individuals are more bowed. In such patients, a short intramedullary rod entering the femur at the apex of the intercondylar notch (femoral anatomical axis I) with a 4-degree distal cutting block (angle $\mathrm{C}$ ) is more likely to produce a distal femoral cut that is perpendicular to the mechanical axis of the femur. However, the choice of the cutting block should be individualized according to the preoperative planning on weight-bearing radiographs of the whole lower extremity. It is also interesting to note that the designers of some instrumentation systems have recommended use of a distal femoral cutting block with a smaller angle in taller individuals on the basis of the assumption that taller patients have a smaller physiological valgus angle of the femur. We cannot confirm this assumption in our study.

Geographical variation in the epidemiology of osteoarthritis has been well documented. The ratio of knee osteoarthritis to hip osteoarthritis is 9:1 for Chinese individuals in Hong Kong ${ }^{4}$, 3:1 for white individuals in the United States ${ }^{1}$, and 1:2 for Swedish individuals ${ }^{2}$. The racial differences in the axial alignment of the lower extremity may contribute to the variation in this ratio. We suspect that the larger knee-joint-obliquity angle in Chinese individuals may contribute to the higher ratio of knee osteoarthritis to hip osteoarthritis among Chinese adults, but additional study is required to confirm this speculation.

\section{References}

1. Ahlberg, A.; Linder, B.; and Binhemd, T. A.: Osteoarthritis of the hip and knee in Saudi Arabia. Internat. Orthop., 14: 29-30, 1990.

2. Danielsson, L., and Hernborg, J.: Morbidity and mortality of osteoarthritis of the knee (gonarthrosis) in Malmo, Sweden. Clin. Orthop., 69: 224-226, 1970.

3. Fang, D.; Chiu, K. Y.; Remedios, I. D.; and Yin, Q.: Osteometry of the Chinese proximal femur. J. Orthop. Surg., 4: 41-45, 1996.

4. Hoaglund, F. T.; Yau, A. C. M. C.; and Wong, W. L.: Osteoarthritis of the hip and other joints in southern Chinese in Hong Kong. Incidence and related factors. J. Bone and Joint Surg., 55-A: 545-557, April 1973. 
5. Hsu, R. W. W.; Himeno, S.; Coventry, M. B.; and Chao, E. Y. S.: Normal axial alignment of the lower extremity and load-bearing distribution at the knee. Clin. Orthop., 255: 215-227, 1990.

6. Hungerford, D. S.; Kenna, R. V.; and Krackow, K. A.: The porous-coated anatomic total knee. Orthop. Clin. North America, 13: 103$122,1982$.

7. Insall, J. N.: Surgical techniques and instrumentation in total knee arthroplasty. In Surgery of the Knee, edited by J. N. Insall, R. E. Windsor, W. N. Scott, M. A. Kelly, and P. Aglietti. Ed. 2, vol. 2, pp. 739-804. New York, Churchill Livingstone, 1993.

8. Jiang, C.-C., and Insall, J. N.: Effect of rotation on the axial alignment of the femur. Pitfalls in the use of femoral intramedullary guides in total knee arthroplasty. Clin. Orthop., 248: 50-56, 1989.

9. Lotke, P. A., and Ecker, M. L.: Influence of positioning of prosthesis in total knee replacement. J. Bone and Joint Surg., 59-A: 77-79, Jan. 1977.

10. Matthewson, M. H., and Dandy, D. J.: Osteochondral fractures of the lateral femoral condyle. A result of indirect violence to the knee. J. Bone and Joint Surg., 60-B(2): 199-202, 1978.

11. Minns, R. S.: Cartilage ulceration and shear fatigue failure [letter]. Lancet, 1: 907-908, 1976.

12. Moreland, J. R.; Bassett, L. W.; and Hanker, G. J.: Radiographic analysis of the axial alignment of the lower extremity. J. Bone and Joint Surg., 69-A: 745-749, June 1987.

13. Ritter, M. A.; Faris, P. M.; Keating, E. M.; and Meding, J. B.: Postoperative alignment of total knee replacement. Its effect on survival. Clin. Orthop., 299: 153-156, 1994.

14. Tomatsu, T.; Imai, N.; Takeuchi, N.; Takahashi, K.; and Kimura, N.: Experimentally produced fractures of articular cartilage and bone: the effects of shear forces on the pig knee. J. Bone and Joint Surg., 74-B(3): 457-462, 1992. 